\title{
Biomechanical analysis of brachial plexus injury: Availability of three-dimensional finite element model of the brachial plexus
}

\author{
ATSUSHI MIHARA $^{1}$, TSUKASA KANCHIKU ${ }^{1}$, NORIHIRO NISHIDA $^{1}$, \\ HARUKI TAGAWA $^{2}$, JUNJI OHGI ${ }^{2}$, HIDENORI SUZUKI ${ }^{1}$, YASUAKI IMAJO ${ }^{1}$, MASAHIRO FUNABA $^{1}$, \\ DAISUKE NAKASHIMA ${ }^{1},{\text { XIAN } \text { CHEN }^{2} \text { and TOSHIHIKO TAGUCHI }}^{2}$ \\ ${ }^{1}$ Department of Orthopedic Surgery, Yamaguchi University Graduate School of Medicine; \\ ${ }^{2}$ Department of Mechanical Engineering, Yamaguchi University, Ube, Yamaguchi 755-8505, Japan
}

Received July 20, 2017; Accepted October 2, 2017

DOI: $10.3892 / \mathrm{etm} .2017 .5607$

\begin{abstract}
Adult brachial plexus injuries frequently lead to significant and permanent physical disabilities. Investigating the mechanism of the injury using biomechanical approaches may lead to further knowledge with regard to preventing brachial plexus injuries. However, there are no reports of biomechanical studies of brachial plexus injuries till date. Therefore, the present study used a complex three-dimensional finite element model (3D-FEM) of the brachial plexus to analyze the mechanism of brachial plexus injury and to assess the validity of the model. A complex 3D-FEM of the spinal column, dura mater, spinal nerve root, brachial plexus, rib bone and cartilage, clavicle, scapula, and humerus were conducted. Stress was applied to the model based on the mechanisms of clinically reported brachial plexus injuries: Retroflexion of the cervical, lateroflexion of the cervical, rotation of the cervical, and abduction of the upper limb. The present study analyzed the distribution and strength of strain applied to the brachial plexus during each motion. When the cervical was retroflexed or lateroflexed, the strain was focused on the C5 nerve root and the upper trunk of the brachial plexus. When the upper limb was abducted, strain was focused on the C7 and $\mathrm{C} 8$ nerve roots and the lower trunk of the brachial plexus. The results of brachial plexus injury mechanism corresponded with clinical findings that demonstrated the validity of this model. The results of the present study hypothesized that the model has a future potential for analyzing pathological conditions of brachial plexus injuries and other injuries or diseases, including that of spine and spinal nerve root.
\end{abstract}

Correspondence to: Dr Norihiro Nishida, Department of Orthopedic Surgery, Yamaguchi University Graduate School of Medicine, 1-1-1 Minami-Kogushi, Ube, Yamaguchi 755-8505, Japan E-mail: nishida3@yamaguchi-u.ac.jp

Key words: brachial plexus injury, finite element model, mechanical property, spine, spinal cord, spinal nerve root

\section{Introduction}

Adult brachial plexus injuries frequently lead to significant physical disabilities. It could be caused by various mechanisms including falls, powerful sports activities, and motor vehicle trauma. Different mechanisms of injury lead to different injury patterns with different levels of plexus damage (1). Since severe injuries often lead to permanent disabilities, prevention of the injury is significant. Investigating the mechanism of the injury by biomechanical approaches should provide aid with regard to preventing injuries.

However, there are no reports of a complex three-dimensional finite element model (3D-FEM) of the spine, dura mater, root, and the brachial plexus all in one model till date. The first objective of this study is to construct a complex 3D-FEM of the spine, dura mater, root, and the brachial plexus. In this study, to assess the validity of the model, we analyzed the patterns of clinically reported brachial plexus injuries by applying stress to the model and verified weather the results of the analysis and clinically reported results correspond. Secondly, we inspected the proposed model's availability in studying the prevention of brachial plexus injury.

\section{Materials and methods}

Model. Using Simpleware ScanIP (Synopsys, Mountain View, CA, USA), a three-dimensional finite element model including the dura mater, vertebrae and intervertebral disk from C2 through Th2, first and second rib bones and costicartilage, right scapula, right clavicle and right humerus was constructed from CT and MRI images of a male human body, supplied by the Visible Human Project (U.S. National Library of Medicine, Bethesda, MD, USA). We constructed the rib bones and costicartilage as the thoracic cage because we believe that the thoracic cage participates in the motion properties of the spine. The reason being that the motion property of the spine and the first rib bone differed between models with or without the thoracic cage and thus it is rational to consider that the model possessing the thoracic cage is considerably likely to reproduce the actual motion of a real human body. We constructed the scapula, clavicle, and humerus to analyze the motion of the upper limb. Since there were no images of the 
brachial plexus, we extended the right spinal nerve roots of C5 to Th1 in proportion to its anatomical form and constructed a model from the root to the trunk as the right brachial plexus. Injuries of the brachial plexus occur predominantly at levels of root to the trunk (2), thus we constructed this level precisely. We extended the trunks of the plexus further to the upper third of the right humerus as a peripheral nerve and fixed the nerves to a rigid plate imitating a muscle. The dura mater was immobilized completely at $\mathrm{C} 2$ level. All components of the model were symmetrically meshed with 20 -node elements. The total number of isoperimetric 20-node elements was 82,011 and the total number of nodes was 285,694 (Fig. 1).

We configured the boundary condition using Jvision (JSOL, Tokyo, Japan). The material constant of each component was derived as follows (Table I) according to previous published studies (3-6). Since the brachial plexus is a continuous component from the spinal nerve root, the mechanical property of the spinal nerve root was used for the modeling of brachial plexus.

Analyzing conditions. We used the LS-DYNA (JSOL) for analyzing. We set four analyzing conditions: Retroflexion of the cervical (case 1), left lateroflexion of the cervical (case 2), left rotation of the cervical (case 3) and abduction of the right upper limb (case 4). These conditions were selected based on mechanisms of brachial plexus injury reported clinically $(1,7)$.

In cases 1-3, the rigid plate, second thoracic vertebrae and lower end of the dura mater were configured at complete constraint. Forced angular displacement was applied to the top of the second cervical vertebrae. The degree of angle applied in each case was defined based on the maximum range of motion of the neck in a human body; $33^{\circ}$ in case $1,23.5^{\circ}$ in case $2,25.5^{\circ}$ in case 3 (8). In the motion of the cervical, the thoracic vertebra do not move because they are immobilized by the thoracic cage. Thus, the rotation center of the forced angular displacement was defined to the center of the bottom of the seventh cervical vertebrae in the three cases.

In case 4 , the spinal column, rib bones, clavicle, scapula, and lower end of the dura mater were configured at complete constraint. Forced angular displacement of $30^{\circ}$ of abduction was applied to the humerus. We configured the angle to be $30^{\circ}$ because over $30^{\circ}$ of abduction, the scapula starts upward rotation, and in this model the scapula is at complete constraint (9). The rotation center was defined to the center of the glenohumeral joint (Fig. 2).

The velocity $(V)$ of the force applied in each case was configured as $10 \mathrm{~km} / \mathrm{hr}$, because motor vehicle accidents, a major factor of brachial plexus injury, occur primarily at velocities lower than $10 \mathrm{~km} / \mathrm{hr}$ in this country. Using the distance from the point of force applied to the rotation center $(r)$, the angular velocity $(w)$ was calculated by the following numerical formula: $V=w r$

Measurement of distribution and size of strain applied to the brachial plexus in each case mentioned above was conducted. There is no knowledge with regard to the threshold value of strain applied to the brachial plexus when symptoms of injuries appear. However, Singh et al have reported that the breaking strain of the dorsal root is $30.8 \pm 8.4 \%$ (10). Galbraith et al have reported via a tension test of an axon of a giant squid that axonal injury of the white matter occurs from strain level of approximately $28 \%$ (11).

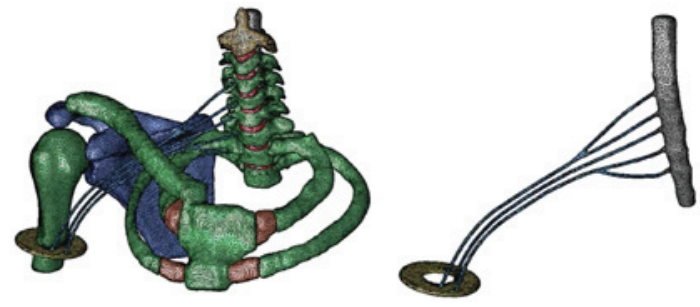

Figure 1. The complex 3D FEM. The spine, dura mater, spinal nerve root, brachial plexus, thoracic cage, and the upper limb was constructed. The spinal nerve roots of $\mathrm{C} 5$ to Th1 was extended in proportion to its anatomical form as the brachial plexus.
A

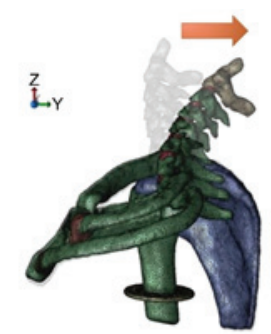

C

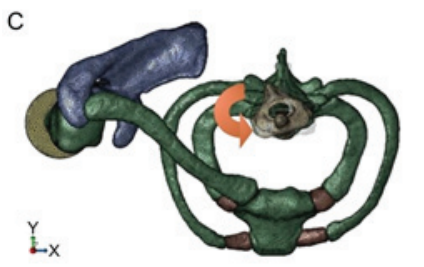

B

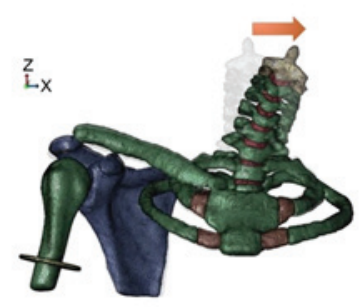

D

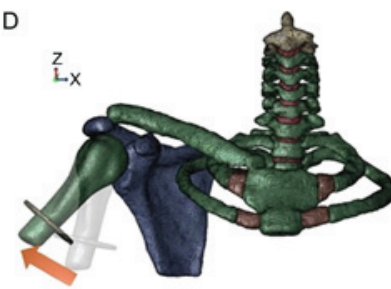

Figure 2. Analyzing conditions. (A) The spine was retroflexed $33^{\circ}$. (B) The spine was lateroflexed $23.5^{\circ}$. (C) The spine was rotated $25.5^{\circ}$. (D) The humerus was abducted $30^{\circ}$.

Thus in this study we assumed the breaking strain of the brachial plexus at $30 \%$.

\section{Results}

Figs. 3-6 illustrates the distribution and size of strain applied to the brachial plexus in each case. In case 1, the strain was focused on the root of C5. Contact of the root and the roof of the intervertebral foramen were observed that led to an increase of strain in one spot. The maximum level of strain was $21 \%$ (Fig. 3). In case 2, the strain was focused on the root of C5 and the upper trunk of the plexus. Lateroflexion of the cervical led to a stretch of the upper trunk of the brachial plexus, applying stress diffusely throughout the upper trunk. The maximum level of strain was $15 \%$ (Fig. 4). In case 3, no focus of strain was observed and the stress was applied diffusely throughout the brachial plexus (Fig. 5). In case 4, strain was focused on the lower trunk of the brachial plexus and the roots of $\mathrm{C} 7$ and $\mathrm{C} 8$. Abduction of the upper limb led to a stretch of the middle and lower trunk, with strain strongly focused in the middle and lower trunk region. The maximum level of strain was 16\% (Fig. 6).

\section{Discussion}

Injuries of the brachial plexus frequently lead to significant physical disabilities, psychological distress, and 
Table I. The mechanical property of each components of the model.

\begin{tabular}{llccr}
\hline Author, Year & \multicolumn{1}{c}{ Component } & Young's modulus & Poisson's ratio & (Refs.) \\
\hline Imajo et al 2009 & Bone & 10,000 & 0.3 & $(3)$ \\
Imajo et al 2009 & Intervertebral disk & 3.4 & 0.4 & $(3)$ \\
Pal 2014 & Costicartilage & 10 & 0.4 & $(4)$ \\
Tencer et al 1985 & Dura mater & 129 & 0.4 & $(5)$ \\
Nishida et al 2015 & Spinal nerve root, Brachial plexus & 2 & 0.4 & $(6)$ \\
\hline
\end{tabular}
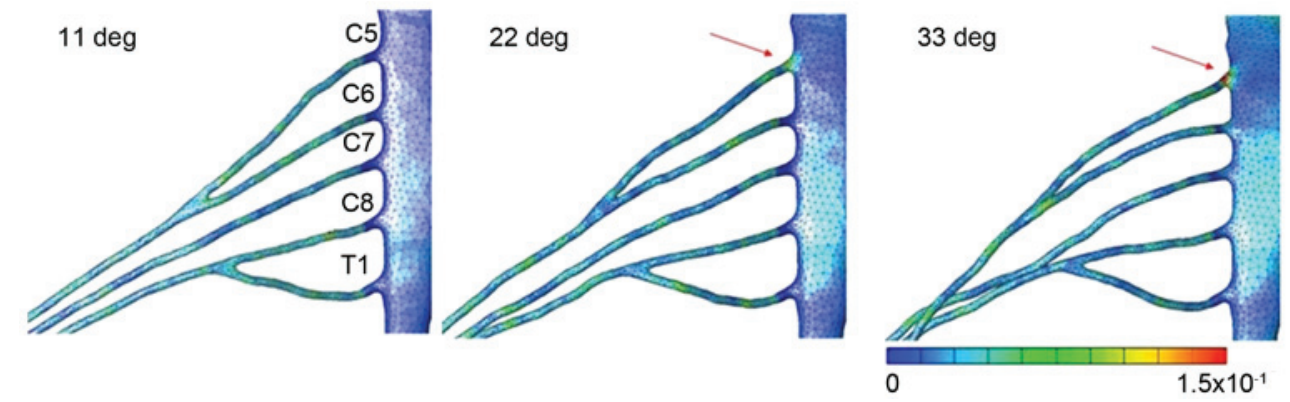

Figure 3. Results for retroflexion of the spine. Strain focused on the root of C5. The maximum strain rate was $21 \%$.
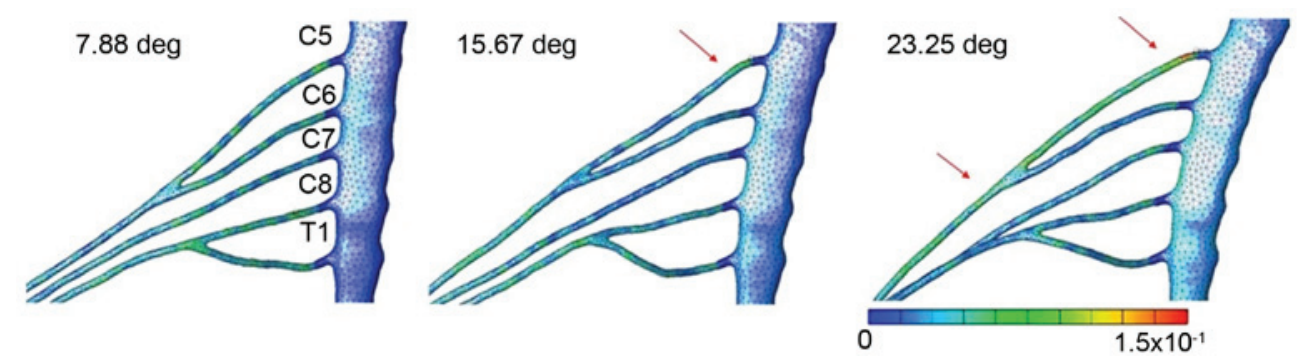

Figure 4. Results for lateroflexion of the spine. Strain focused on the root of C5 and the upper trunk of the plexus. The maximum strain rate was $15 \%$.

socioeconomic hardship. Excluding brachial plexus injuries in infants during delivery, adult brachial plexus injuries predominantly occur in young men or boys with an average age of 20 to 30 years $(1,2,12-14)$. Injuries could be caused by various mechanisms, including penetrating injuries, falls, powerful sports activity, and motor vehicle accidents. In closed injury, pathological types of injury include avulsion of the nerve at root level or rupture or significant stretch at various levels of the brachial plexus $(1,7)$.

Doi et al have reported successful outcomes with double free muscle transfer against complete avulsion of the brachial plexus (14). Bertelli et al have reported satisfactory results in abduction of the shoulder with spinal accessory nerve transfer to the suprascapular nerve in patients with complete brachial plexus injury (15). However, even though several surgical treatment options exist, perfect recovery after complete brachial plexus injury is impossible and physical difficulty is inevitable even after surgery (14-16). Therefore, prevention of the injury becomes important. Analyzing the mechanism of injury could provide further knowledge for preventing the injury.

Considerable number of brachial plexus injuries are caused by closed trauma $(77-91 \%)(12,17)$. Nerve injury in these cases is from traction and compression, with traction accounting for $95 \%$ of closed injuries (1). Following traction, any combination of avulsion, rupture, or significant stretch might occur throughout the brachial plexus. However, certain patterns of injuries seem to be substantially prevalent. The supraclavicular region is affected considerably frequently $(70-91 \%)$ than the retroclavicular or infraclavicular regions $(1,2)$. The roots and trunks are considerably commonly injured compared to the divisions, cords, or branches. Clinically, traction to the brachial plexus occurs when the head and neck are violently moved away from the ipsilateral shoulder or when the upper limb is abducted violently over the head with significant force. The former results in injury to the upper elements of the brachial plexus (C5, C6 roots or upper trunk), and the latter results in injury to the lower elements (C8, T1 roots or lower trunk) $(1,7,13)$. Similar results were reported in cadaver experiments (7). It is assumed that panplexal injuries occur when the force of injury is significantly high. Bertelli et al reported the frequency of panplexal injury, upper brachial plexus injury, and lower brachial plexus injury within supraclavicular injuries were 50,47 , and $3 \%$ respectively (2). 

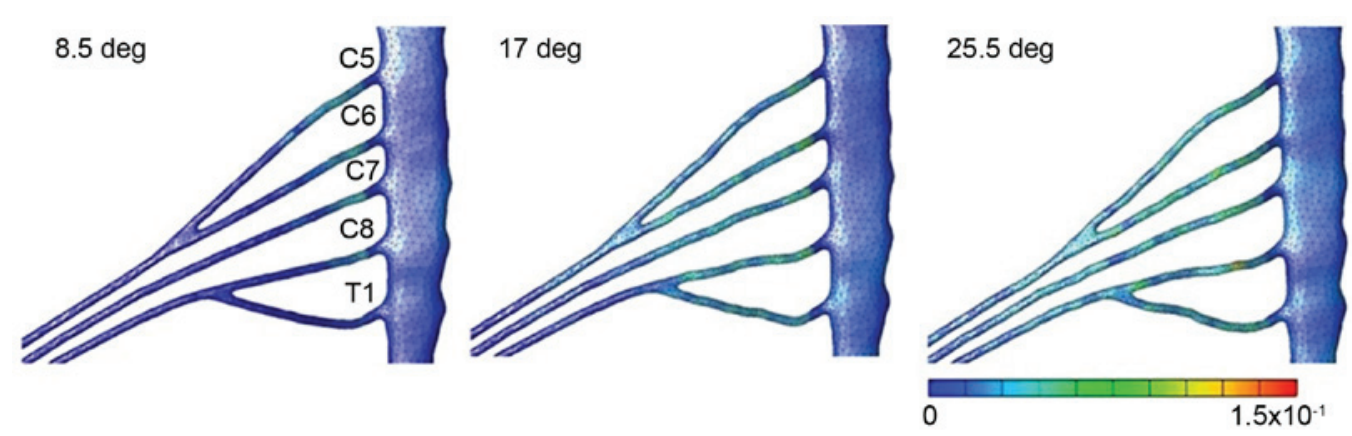

Figure 5. Results for rotation of the spine. No focus of strain was observed and the stress was applied diffusely throughout the brachial plexus.
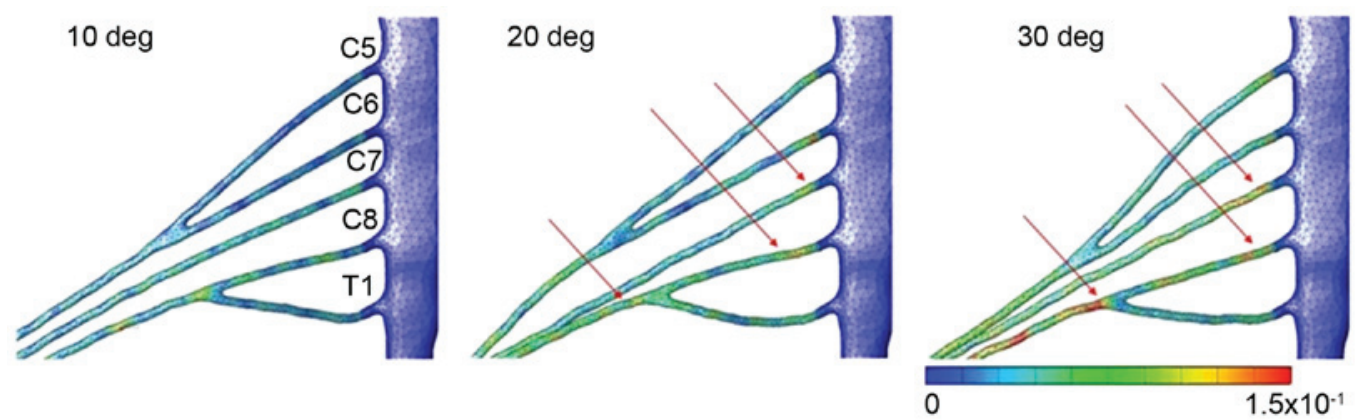

Figure 6. Results for abduction of the humerus. Strain focused on the lower trunk of the brachial plexus and the roots of C7 and C8. The maximum strain rate was $16 \%$.

Based on this prior knowledge, we conducted stress analysis using 3D-FEM of the brachial plexus. We conducted four patterns of stress based on the patterns of mechanisms of injury described clinically. Nishida et al have reported a 3D-FEM of the spinal cord to conduct stress analysis of various clinical conditions, such as ossification of the posterior longitudinal ligament (OPLL) and cervical spondylotic myelopathy (CSM) $(18,19)$. Imajo et al have constructed 3D-FEM of the spinal column in different studies (3). However, there are no reports of a complex 3D-FEM of the spine, dura mater, root, and the brachial plexus all in one model till date. The overall objective of this study was to develop a complex 3D-FEM of the spine, dura mater, root, brachial plexus, and other components that stimulate the clinical situation appropriately.

In previous studies of 3D-FEM of the spinal cord by Nishida et al and Kato et al, the mechanical property of a bovine spinal cord was used in the model since it was impossible to obtain fresh human spinal cord (18-20). Li and Dai noted that it was reasonable to employ the mechanical properties of bovine spinal cord because the brain and spinal cord of cattle and humans demonstrate similar injury changes (21). In this study, for the spinal nerve root, we used the mechanical property of a porcine nerve root because it was impossible to obtain fresh human spinal nerve root and there were no previous reports that obtained mechanical property of a bovine spinal nerve root. We believe this is reasonable because Olmarker et al reported that human and porcine spinal nerve roots demonstrated significant resemblance in both neural and vascular anatomy (22).

In our analysis results, retroflexion and lateroflexion of the cervical, simulating a clinical situation of moving the head and neck away from the shoulder, resulted in the focus of strain in the upper trunk and the roots of C5 and C6. From these results, traction of upper region of the brachial plexus is suggested that corresponds to the clinical findings as reported. Abduction of the upper limb resulted in focus of strain in the lower trunk and the roots of $\mathrm{C} 7$ and $\mathrm{C} 8$. These results, suggesting traction of lower region, also corresponds to clinical findings. Rotation of the cervical resulted in no focus of strain. We believe this result is appropriate because rotation of the neck applies less force of traction to the nerves compared to the other three situations. Overall, we believe that the validity of this model was demonstrated. From our results, discussing approaches of protection from strong force leading to retroflexion or lateroflexion of the neck, or abduction of the upper limb might be acceptable strategies of preventing brachial plexus injuries.

There are certain limitations in this study. Firstly, since there is no knowledge of threshold value of strain when symptoms of brachial plexus injury appear, a quantitative evaluation using the model was impossible. Thus, this is a qualitative study. Subsequently, we could not evaluate upper limb abduction further than $30^{\circ}$. To express further abduction, we require reproducing the upward rotation of the scapula and the motion of other components of the shoulder which is a future task of this model. Reproducing other tissues around the brachial plexus and the cervical spine, such as muscles and vessels might render the model considerably precise and substantially accurate results might be expected. Finally, the lack of assessment of blood flow of the nerves is a major limitation, because loss of blood flow could be a cause of neurological disorder. Significant further tests might include examination of a considerably complicated combination of motion of the cervical and the upper limb and applying further stress to 
examine the type of motion that leads to panplexal brachial plexus injury with the smallest amount of stress.

Although certain limitations are mentioned above, overall in this study, we were able to illustrate the pathology of brachial plexus injuries with this complex 3D-FEM. The results of the analysis supported the conventional clinical reports. We believe that this model has a potential of being used in various analysis in the future, including studies to prevent brachial plexus injuries.

We constructed a complex 3D-FEM of the spine, dura mater, spinal nerve root, and the brachial plexus all in one model. Retroflexion and lateroflexion of the cervical resulted in upper region brachial plexus injury and abduction of the upper limb resulted in lower region brachial plexus injury.

We were able to illustrate certain pathology of brachial plexus injury, demonstrating the validity of this model. There is a future potential of using this model for analyzing pathological conditions including the spine and spinal nerve roots.

\section{Acknowledgements}

The authors express their gratitude to the cooperation provided by the members of the Medical and Mechanical Engineering Laboratory of Yamaguchi University Graduate School, and graduate students from this Mechanical Engineering Laboratory.

\section{References}

1. Moran SL, Steinmann SP and Shin AY: Adult brachial plexus injuries: Mechanism, patterns of injury, and physical diagnosis. Hand Clin 21: 13-24, 2005.

2. Bertelli JA, Ghizoni MF and Soldado F: Patterns of brachial plexus stretch palsy in a prospective series of 565 surgically treated patients. J Hand Surg Am 42: 443-446.e2, 2017.

3. Imajo Y, Hiiragi I, Kato Y and Taguchi T: Use of the finite element method to study the mechanism of spinal cord injury without radiological abnormality in the cervical spine. Spine (Phila Pa 1976) 34: E83-E87, 2009.

4. Pal S: Mechanical Properties of Biological Materials. In: Design of Artificial Human Joints \& Organs. 1st edition. Springer, New York, NY, pp23-40, c2014.

5. Tencer AF, Allen BL Jr and Ferguson RL: A biomechanical study of thoracolumbar spine fractures with bone in the canal. Part III. Mechanical properties of the dura and its tethering ligaments. Spine (Phila Pa 1976) 10: 741-747, 1985

6. Nishida N, Kanchiku T, Ohgi J, Ichihara K, Chen X and Taguchi T: Mechanical properties of nerve roots and rami radiculares isolated from fresh pig spinal cords. Neural Regen Res 10: 1869-1873, 2015.
7. Coene LN: Mechanisms of brachial plexus lesions. Clin Neurol Neurosurg 95 (Suppl): S24-S29, 1993

8. White AA III and Panjabi MM: The basic kinematics of the human spine. A review of past and current knowledge. Spine (Phila Pa 1976) 3: 12-20, 1978.

9. Inman VT, Saunders JB and Abbott LC: Observations on the function of the shoulder joint. 1944. Clin Orthop Relat Res: 3-12, 1996.

10. Singh A, Lu Y, Chen C and Cavanaugh JM: Mechanical properties of spinal nerve roots subjected to tension at different strain rates. J Biomech 39. 1669-1676, 2006.

11. Galbraith JA, Thibault LE and Matteson DR: Mechanical and electrical responses of the squid giant axon to simple elongation. J Biomech Eng 115: 13-22, 1993.

12. Dubuisson AS and Kline DG: Brachial plexus injury: A survey of 100 consecutive cases from a single service. Neurosurgery 51: 673-683, 2002.

13. Soldado F, Ghizoni MF and Bertelli J: Injury mechanisms in supraclavicular stretch injuries of the brachial plexus. Hand Surg Rehabil 35: 51-54, 2016

14. Doi K, Muramatsu K, Hattori Y, Otsuka K, Tan SH, Nanda V and Watanabe M: Restoration of prehension with the double free muscle technique following complete avulsion of the brachial plexus. Indications and long-term results. J Bone Joint Surg Am 82: 652-666, 2000.

15. Bertelli AJ and Ghizoni MF: Results of spinal accessory to suprascapular nerve transfer in 110 patients with complete palsy of the brachial plexus. J Neurosurg Spine 24: 990-995, 2016.

16. Liu Y, Lao J, Gao K, Gu Y and Zhao X: Functional outcome of nerve transfers for traumatic global brachial plexus avulsion. Injury 44: 655-660, 2013.

17. Songcharoen P: Brachial plexus injury in Thailand: A report of 520 cases. Microsurgery 16: 35-39, 1995.

18. Nishida N, Kato Y, Imajo $\mathrm{Y}$, Kawano $\mathrm{S}$ and Taguchi $\mathrm{T}$ : Biomechanical analysis of cervical spondylotic myelopathy: The influence of dynamic factors and morphometry of the spinal cord. J Spinal Cord Med 35: 256-261, 2012.

19. Nishida N, Kanchiku T, Kato Y, Imajo Y, Yoshida Y, Kawano S and Taguchi T: Biomechanical analysis of cervical myelopathy due to ossification of the posterior longitudinal ligament: Effect of posterior decompression and kyphosis following decompression. Exp Ther Med 7: 1095-1099, 2014.

20. Kato Y, Kataoka H, Ichihara K, Imajo Y, Kojima T, Kawano S, Hamanaka D, Yaji $K$ and Taguchi $T$ : Biomechanical study of cervical flexion myelopathy using a three-dimentional finite element method. J Neurosurg Spine 8: 436-441, 2008.

21. Li XF and Dai LY: Three-dimensional finite element model of the cervical spinal cord: Preliminary results of injury mechanism analysis. Spine (Phila Pa 1976) 34: 1140-1147, 2009.

22. Olmarker K, Holm S, Rosenqvist AL and Rydevik B: Experimental nerve root compression. A model of acute, graded compression of the porchine cauda equine and an analysis of neural and vascular anatomy. Spine (Phila Pa 1976) 16: 61-69, 1991.

This work is licensed under a Creative Commons

Attribution-NonCommercial-NoDerivatives 4.0 International (CC BY-NC-ND 4.0) License. 